\title{
Thermoreversible hydrogel scaffolds for articular cartilage engineering
}

\author{
John P. Fisher, ${ }^{1 *}$ Seongbong Jo, ${ }^{2}$ Antonios G. Mikos, ${ }^{2}$ A. Hari Reddi ${ }^{1}$ \\ ${ }^{1}$ Department of Orthopaedic Surgery, Center for Tissue Regeneration and Repair, School of Medicine, University of \\ California, Davis, Research Building I, Room 2000, 4635 Second Avenue, Sacramento, California 95817 \\ ${ }^{2}$ Department of Bioengineering, Rice University, P.O. Box 1892, MS 142, Houston, Texas 77251
}

Received 18 December 2003; revised 18 June 2004; accepted 22 June 2004

Published online 14 September 2004 in Wiley InterScience (www.interscience.wiley.com). DOI: 10.1002/jbm.a.30148

\begin{abstract}
Articular cartilage has limited potential for repair. Current clinical treatments for articular cartilage damage often result in fibrocartilage and are associated with joint pain and stiffness. To address these concerns, researchers have turned to the engineering of cartilage grafts. Tissue engineering, an emerging field for the functional restoration of articular cartilage and other tissues, is based on the utilization of morphogens, scaffolds, and responding progenitor/stem cells. Because articular cartilage is a water-laden tissue and contains within its matrix hydrophilic proteoglycans, an engineered cartilage graft may be based on synthetic hydrogels to mimic these properties. To this end, we have developed a polymer system based on the hydrophilic copolymer poly(propylene fumarate-co-ethylene glycol) $[\mathrm{P}(\mathrm{PF}-\mathrm{co}-\mathrm{EG})]$. Solutions of this polymer are liquid below $25^{\circ} \mathrm{C}$ and gel above $35^{\circ} \mathrm{C}$, allowing an aqueous solution containing cells at room temperature to form a hydrogel with encapsulated cells at physiological body temperature. The objective of this work was to determine the effects of the
\end{abstract}

hydrogel components on the phenotype of encapsulated chondrocytes. Bovine articular chondrocytes were used as an experimental model. Results demonstrated that the components required for hydrogel fabrication did not significantly reduce the proteoglycan synthesis of chondrocytes, a phenotypic marker of chondrocyte function. In addition, chondrocyte viability, proteoglycan synthesis, and type II collagen synthesis within P(PF-co-EG) hydrogels were investigated. The addition of bone morphogenetic protein-7 increased chondrocyte proliferation with the P(PF-co-EG) hydrogels, but did not increase proteoglycan synthesis by the chondrocytes. These results indicate that the temperatureresponsive $\mathrm{P}(\mathrm{PF}-\mathrm{co}$-EG) hydrogels are suitable for chondrocyte delivery for articular cartilage repair. () 2004 Wiley Periodicals, Inc. J Biomed Mater Res 71A: 268-274, 2004

Key words: cartilage; chondrocytes; morphogens; hydrogels; tissue engineering

\section{INTRODUCTION}

Native articular cartilage is a durable long-lasting tissue, with an extracellular matrix consisting mostly of proteoglycans and type II collagen. Cartilage matrix proteoglycans, most notably aggrecan, are negatively charged molecules that induce both a repelling force, due to charged proteoglycans repulsion of negatively, and an osmotic swelling force, as water moves into the tissue to ensure electroneutrality. ${ }^{1}$ The resulting stress that is induced in articular cartilage allows the tissue

*Present address: Department of Chemical Engineering, University of Maryland, College Park, MD 20742

Correspondence to: A. H. Reddi; e-mail: ahreddi@ucdavis. edu

Contract grant sponsor: Lawrence Ellison Chair Endowment for Musculoskeletal Molecular Biology and Regenerative Medicine

Contract grant sponsor: National Institutes of Health; contract grant number: R01-AR48756

(C) 2004 Wiley Periodicals, Inc. to withstand large loads by virtue of the matrix's tensile strength and the exudable water held within the tissue. As a result, articular cartilage possesses significant mechanical properties, with a compressive modulus of $0.79 \mathrm{MPa}$, a shear modulus of $0.69 \mathrm{MPa}$, and a tensile modulus varying between 0.3 and 10.2 $\mathrm{MPa} .^{2}$ Nevertheless, mechanical loads may be applied to articulating joints with such great magnitude and swift rate, that cartilage is not able to accept the force and thus fails by either surface or both surface and subchondral bone disruption.

The surgical options for articular cartilage repair typically include graft implantation and subchondral bone microfractures. Grafting procedures entail the implantation of autologous osteochondral plugs, allografts, periosteal grafts, and perichondrial grafts. ${ }^{3}$ Subchondral bone penetration procedures include subchondral drilling, microfracture, and spongialization. Thus, although both procedures rely on growth factors, differentiated cells, and pluripotent mesenchymal cells to repair the damaged site, the grafting 
strategies implant these participants directly, whereas subchondral bone penetration methods initiate their migration from the underlying bone. ${ }^{4}$ Unfortunately, the clinical outcomes of these treatments often result in the formation of fibrocartilage and are associated with joint pain and stiffness. The inadequacies of current treatments for articular cartilage defects have led us and others to consider the development of articular cartilage grafts by following the principles of tissue engineering.

The properties of an ideal engineered articular cartilage graft include biocompatibility, mechanical strength, and implantability. Furthermore, the graft typically would contain encapsulated chondrocytes for the reconstruction of the damaged tissue matrix, and therefore must be able to deliver a viable and functional cell population. Thus, the graft must not disturb the chondrocyte's native phenotype. It was shown more than 20 years ago that the phenotype of chondrocytes includes a rounded cell morphology, type II collagen synthesis, and glycosaminoglycan synthesis. ${ }^{5,6}$ This phenotype can be maintained in vitro by controlling cell shape, either by supplementing the media with growth factors or through physical means, such as high-density culture or, most relevant to this discussion, suspension culture. ${ }^{5,6}$ In addition, the graft must also be permeable enough to allow for cell nourishment, but dense enough to maintain its shape and provide structural support both to the surrounding cartilage and the subchondral bone. Finally, such a graft would be degradable, so as to leave uninterrupted, regenerated articular cartilage tissue.

The strategy that we seek to develop for the treatment of articular cartilage defects involves the injection of a chondrocyte suspension into the defect site. The chondrocytes would be initially held within a solution containing the temperature-sensitive, poly(propylene fumarate-co-ethylene glycol) [P(PF-co-EG)] copolymer which, when exposed to the in vivo $37^{\circ} \mathrm{C}$ environment, forms a hydrogel by the association of the copolymer chains. This study explores the efficacy of this system. Specifically, we seek to determine the effects of the hydrogel components and hydrogel suspension culture on chondrocyte phenotype, as well as bone morphogenetic protein-7 (BMP-7) augmentation of chondrocyte phenotype within hydrogel suspension culture. ${ }^{7}$

\section{MATERIALS AND METHODS}

\section{Chondrocyte isolation and culture}

Fresh cartilage was harvested from the metatarsal phalangeal joint of calves, washed three times in Dulbecco's modified Eagle medium (DMEM)/F12 (1:1) media (Gibco, Carlsbad, CA), and digested in a $0.2 \%$ collagenase P (Sigma-
Aldrich, St. Louis, MO) DMEM/F12 (1:1) media solution overnight at $37^{\circ} \mathrm{C}$. Released chondrocytes were then filtered with a 70- $\mu \mathrm{m}$ nylon mesh, washed three times in media with $10 \%$ fetal bovine serum (Gibco), and then allowed to recover from the enzyme digestion for $2 \mathrm{~h}$ in serum-supplemented media. Standard culture conditions included DMEM/F12 media with $50 \mu \mathrm{g} / \mathrm{mL}$ ascorbic-2-phosphate (Sigma-Aldrich), $1 \mathrm{mg} / \mathrm{mL}$ bovine serum albumin (Sigma-Aldrich), 1.2 $\mathrm{mg} / \mathrm{mL}$ sodium bicarbonate (Sigma-Aldrich), 1\% penicillin/ streptomycin (Gibco), and 10\% fetal bovine serum (Gibco). In some cultures, BMP-7 (a gift from Dr. David Rueger, Creative Biomolecules) was also added to the culture media at concentrations ranging from 10 to $100 \mathrm{ng} / \mathrm{mL}$. Cell viability was assessed using methylthiazolyldiphenyl-tetrazolium bromide (MTT, Sigma-Aldrich), where $200 \mu \mathrm{L}$ of $5 \mathrm{mg}$ $\mathrm{MTT} / \mathrm{mL}$ phosphate-buffered saline was added to the culture and incubated for $2 \mathrm{~h}$. MTT stain intensity was quantified using National Institutes of Health Image software.

\section{Copolymer synthesis and hydrogel fabrication}

P(PF-co-EG) was synthesized by the copolymerization of poly(propylene fumarate) $(2000 \mathrm{~g} / \mathrm{mol})$, itself a polyester synthesized from diethyl fumarate (Acros, Fairlawn, NJ) and propylene glycol (Acros), and methoxy poly(ethylene glycol) (550 g/mol, Sigma-Aldrich), as previously described. ${ }^{8,9}$ $\mathrm{P}$ (PF-co-EG) hydrogels were formed by first dissolving the copolymer $(0.05 \mathrm{~g} / \mathrm{mL})$ and glycerol $(0.3 \mathrm{~mL} / \mathrm{mL})$ into DMEM/F12 media; the solution was subsequently brought to $\mathrm{pH}$ 7.4. When placed in a $37^{\circ} \mathrm{C}$ environment, the solution formed a thin hydrogel. All cultures were performed with $10 \times 10^{6}$ chondrocytes per milliliter of copolymer solution.

To form a P(PF-co-EG) hydrogel containing bovine chondrocytes, the procedure indicated above was first performed so as to form a cell-free hydrogel on the bottom of the tissue culture polystyrene (TCPS) dish; this was necessary to ensure that the chondrocytes did not attach to the TCPS. Next, chondrocytes were suspended within the copolymer/media solution and then added above the cell-free P(PF-co-EG) hydrogel. After incubation, a hydrogel with encapsulated chondrocytes was formed. To remove the copolymer hydrogel from the constructs for the assays described below, the construct was placed in a $4^{\circ} \mathrm{C}$ refrigerator for $10 \mathrm{~min}$. The reduced temperature environment allows the temperaturesensitive hydrogel to dissolve.

\section{Control hydrogel constructs}

Chondrocytes were cultured in agarose and alginate gels as control groups. For agarose hydrogels, a $4 \%$ agarose (BioRad, Hercules, CA) aqueous mixture was autoclaved to form an aqueous agarose solution. One milliliter of the pure agarose solution, warmed to $37^{\circ} \mathrm{C}$, was added to the TCPS dish and subsequently refrigerated for 10-15 min to allow the solution to gel. A cell-free hydrogel layer is required so that hydrogel-encapsulated chondrocytes do not attach to the underlying TCPS. Next, a separate agarose solution was added to pelleted chondrocytes, the chondrocytes were suspended within this agarose solution, and then the suspen- 
TABLE I

Primer Sequences for Quantitative RT-PCR Assays

\begin{tabular}{lll}
\hline \multicolumn{1}{c}{ Protein } & \multicolumn{1}{c}{ Segment } & \multicolumn{1}{c}{ Sequence } \\
\hline Type II collagen & Forward primer & Reverse primer \\
& Probe & GCATTGCCTACCTGGACGAA \\
Fibronectin & Forward primer & CGTTGGAGCCCTGGATA \\
& Reverse primer & TGCTGGCAACCTCAAGAGGCTCTG \\
GAPDH & Probe & GGCATTGATGAAGAACCCTTACAG \\
& Forward primer & GGTAGGCCGTCAAGGT \\
& Reverse primer & CCGAGTCCTGGAACCTCTGCTAGTGC \\
& Probe & GGCGCCAAGAGGGCAT \\
& & GTGGTTCACGCCCATCACA \\
\end{tabular}

sion was added above the cell-free agarose hydrogel. This agarose solution containing chondrocytes was then gelled by refrigeration. Finally, for the assays described below, the agarose hydrogel was removed from the construct by physical disruption.

For the alginate hydrogels, a $1.2 \%$ alginate (Sigma-Aldrich) aqueous solution with $0.15 \mathrm{M} \mathrm{NaCl}$ and $0.025 \mathrm{M}$ HEPES (Sigma-Aldrich) was first created ( $\mathrm{pH} 7.4)$. This solution was then autoclaved and subsequently filter sterilized. One milliliter of the alginate solution was added to the TCPS dish and gelled by the addition of $0.15 \mathrm{~mL}$ of $0.10 \mathrm{M}$ $\mathrm{CaCl}_{2}$ (Sigma-Aldrich). The gel was allowed to set for 10-15 $\mathrm{min}$. This cell-free hydrogel layer is required so that hydrogel-encapsulated chondrocytes do not attach to the underlying TCPS. Next, a separate alginate solution was added to pelleted chondrocytes, the chondrocytes were suspended within this alginate solution, and the suspension was added above the cell-free alginate hydrogel. This alginate solution containing chondrocytes was also gelled with the addition of $0.15 \mathrm{~mL}$ of $0.10 \mathrm{M} \mathrm{CaCl}_{2}$. Finally, the addition of $0.5 \mathrm{~mL}$ of 1.0 $\mathrm{m} M$ ethylenediaminetetraacetic acid allowed for easy removal of the alginate hydrogel from the construct for the assays described below.

All cultures were performed with $10 \times 10^{6}$ chondrocytes per milliliter of either agarose or alginate.

\section{Proteoglycan synthesis assay}

Proteoglycan synthesis was assessed by measuring the amount of radioactive ${ }^{35} \mathrm{~S}$ incorporated into matrix proteins by cultured chondrocytes. First, $40 \mu \mathrm{Ci} / \mathrm{mL}{ }^{35} \mathrm{~S}$ (PerkinElmer, Wellesley, MA) in the form of sodium sulfate was added to the chondrocyte culture and incubated for $4 \mathrm{~h}$. Free ${ }^{35} \mathrm{~S}$ was removed by phosphate-buffered saline washes and then $10 \%$ serum-supplemented DMEM/F12 media was added to the culture. After disruption of the sample (as described above), the sample and media were centrifuged. The supernate was removed by aspiration and the sample incubated in a proteinase $\mathrm{K}$ solution (Sigma-Aldrich, 10 $\mathrm{mg} / 12 \mathrm{~mL}$ TE buffer) overnight at $56^{\circ} \mathrm{C}$. After digestion, a $200-\mu \mathrm{L}$ sample, followed by $1.5 \mathrm{~mL}$ of elution buffer $(4 \mathrm{M}$ guanidine $\mathrm{HCl}, 0.050 \mathrm{M}$ sodium acetate, $0.5 \%$ Triton- $\mathrm{X}$; all Sigma-Aldrich), was added to a Sephadex column. The eluate, which contains ${ }^{35} \mathrm{~S}$ incorporated into primarily sulfated glycosaminoglycans, was collected from the column and 18 $\mathrm{mL}$ of scintillation cocktail was added to the eluted sample, which was subsequently read with a LS 6500 Scintillation
Counter (Beckman Coulter, Fullerton, CA). Radioactivity was normalized to DNA content, which was determined by a picogreen DNA assay (Molecular Probes, Eugene, OR).

\section{RNA isolation}

Total RNA was isolated from experimental samples using a RNeasy Mini Kit (Qiagen, Valencia, CA). Initially, experimental samples were collected and the hydrogel matrix was removed as described above. Following established procedures, the chondrocytes were lysed and the DNA was digested using a 15-min RNase-free DNase I treatment (Qiagen). The RNA was then isolated and collected in RNasefree water.

\section{Quantitative reverse transcriptase polymerase chain reaction (RT-PCR)}

Quantitative RT-PCR was performed using a TaqMan EZ RT-PCR kit (Applied Biosystems, Foster City, CA) as well as oligonucleotide primers and probes for type II collagen, fibronectin, and GAPDH control (Applied Biosystems). The primer and probe sequences are detailed in Table I. The reaction was conducted on an ABI Prism 7700 sequence detector (Applied Biosystems), using thermal cycling conditions of $2 \mathrm{~min}$ at $50^{\circ} \mathrm{C}, 30 \mathrm{~min}$ at $60^{\circ} \mathrm{C}, 5 \mathrm{~min}$ at $95^{\circ} \mathrm{C}$, and 40 cycles of $20 \mathrm{~s}$ at $94^{\circ} \mathrm{C}$ and $1 \mathrm{~min}$ at $62^{\circ} \mathrm{C}$. Each RNA sample was studied in triplicate for each mRNA of interest.

\section{Statistical analysis}

Sets of data were first inspected with an F-test for treatments. ${ }^{10}$ The null hypothesis, that the means of each set were equal, was evaluated with a 95\% confidence level $(\alpha=$ $0.05)$. If the null hypothesis was found to be false, indicating that the means of the different experimental treatment sets were not equal, then Tukey's multiple comparison test was performed. ${ }^{11}$ Tukey's multiple comparison test then indicated, in a pair-wise manner, the relationship between the different sets. 


\section{RESULTS}

Initial studies examined the effects of P(PF-co-EG) and glycerol addition to culture media upon the subsequent $4 \mathrm{~h}$ of proteoglycan synthesis by freshly isolated chondrocytes cultured in monolayer on TCPS [Fig. 1(A)]. The addition of P(PF-co-EG) was studied at concentrations varying from 0.1 to $5.0 \mathrm{wt} \%$, where 5.0 wt \% copolymer is used in the fabrication of the P(PFco-EG) hydrogels. Similarly, the addition of glycerol was studied at concentrations varying from 1 to $30 \mathrm{vol}$ $\%$, where $30 \mathrm{vol} \%$ glycerol is used in hydrogel fabrication. Results showed that chondrocytes are viable in

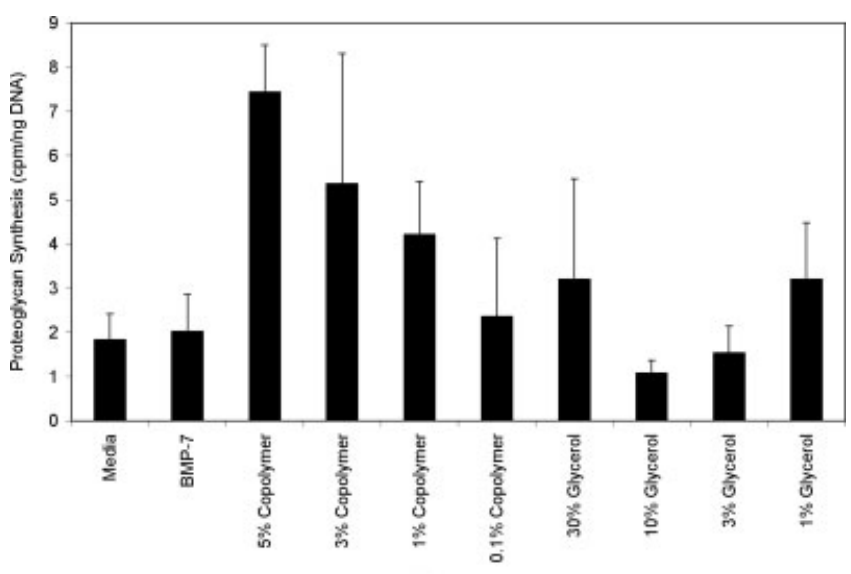

(A)

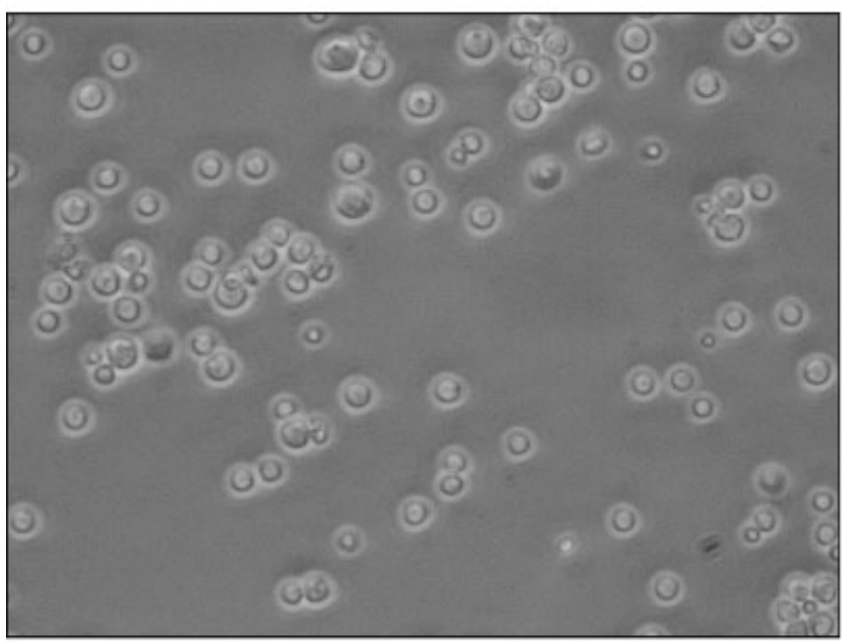

(B)

Figure 1. (A) The effect of $\mathrm{P}(\mathrm{PF}-\mathrm{co}-\mathrm{EG})$ hydrogel components, namely, the $\mathrm{P}$ (PF-co-EG) copolymer and glycerol, on the proteoglycan synthesis of chondrocytes cultured in monolayer for $4 \mathrm{~h}$ on TCPS. Results showed that the general trends of increasing copolymer concentration and decreasing glycerol concentrations result in an increase in proteoglycan synthesis. Only the highest concentration of copolymer (5 wt \%) showed a statistically significant increase in proteoglycan synthesis over the normal media and BMP-7augmented media controls. The mean and standard deviation $(n=3)$ are reported. (B) Micrograph of chondrocytes cultured in monolayer with 30 wt \% glycerol.

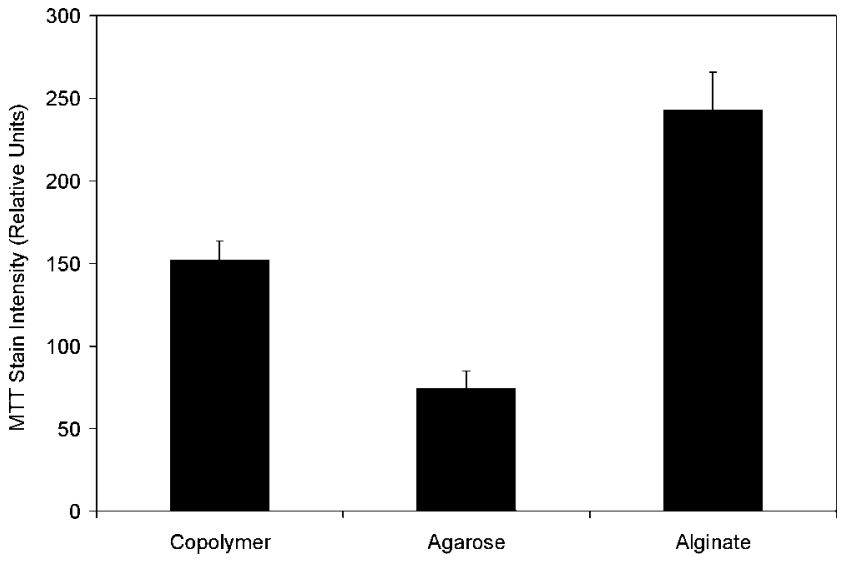

Figure 2. Chondrocyte proliferation as assessed by MTT stain intensity, in the experimental $\mathrm{P}(\mathrm{PF}-\mathrm{co}-\mathrm{EG})$ copolymer hydrogels as well as the control alginate and agarose hydrogels after 8 days of culture. Chondrocyte proliferation within the $\mathrm{P}(\mathrm{PF}-\mathrm{Co}-\mathrm{EG})$ hydrogels was found to be at an intermediate level between the two control groups. The mean and standard deviation $(n=3)$ are reported.

media augmented with up to 5 wt \% copolymer and $30 \mathrm{vol} \%$ glycerol [Fig. 1(A,B)]. Results also indicated a general trend of increasing proteoglycan synthesis with increasing $\mathrm{P}$ (PF-co-EG) copolymer concentration in the culture media. Alternatively, a general trend of decreasing proteoglycan synthesis was observed when glycerol concentration was increased from 1 to $10 \mathrm{vol} \%$. Although the mean proteoglycan synthesis in $30 \mathrm{vol} \%$ glycerol cultures was higher than that in the $10 \mathrm{vol} \%$ cultures, it was not significantly different than in any of the other glycerol cultures. Only the highest concentration of copolymer ( $5 \mathrm{wt} \%)$ showed a statistically significant increase in proteoglycan synthesis over the normal media and BMP-7-augmented media control groups $(p<0.0003)$. The addition of BMP-7 to the DMEM/F12 media did not significantly increase proteoglycan synthesis over the unaugmented-media control culture during the 4-h incubation.

Further studies examined bovine articular chondrocytes at days 1 and 8 when cultured within P(PF-coEG), agarose, and alginate hydrogels. Results showed that chondrocyte proliferation after 8 days of culture within $\mathrm{P}(\mathrm{PF}-\mathrm{co}-\mathrm{EG})$ hydrogels was at a level between that in alginate and agarose hydrogels (Fig. 2). However, proteoglycan synthesis was significantly lower in $\mathrm{P}$ (PF-co-EG) hydrogels than in the alginate and agarose hydrogels after both 1 and 8 days of culture [Fig. 3(A)]. The addition of BMP-7 did not significantly alter the level of proteoglycan synthesis in any case [Fig. 3(B)]. Furthermore, chondrocytic expression of extracellular matrix proteins was studied in these hydrogels, showing that chondrocytes cultured within $\mathrm{P}(\mathrm{PF}-\mathrm{co}-\mathrm{EG})$ produced $25 \%$ of the type II collagen and $44 \%$ of the fibronectin produced by chondrocytes cultured in agarose hydrogels (Fig. 4). 


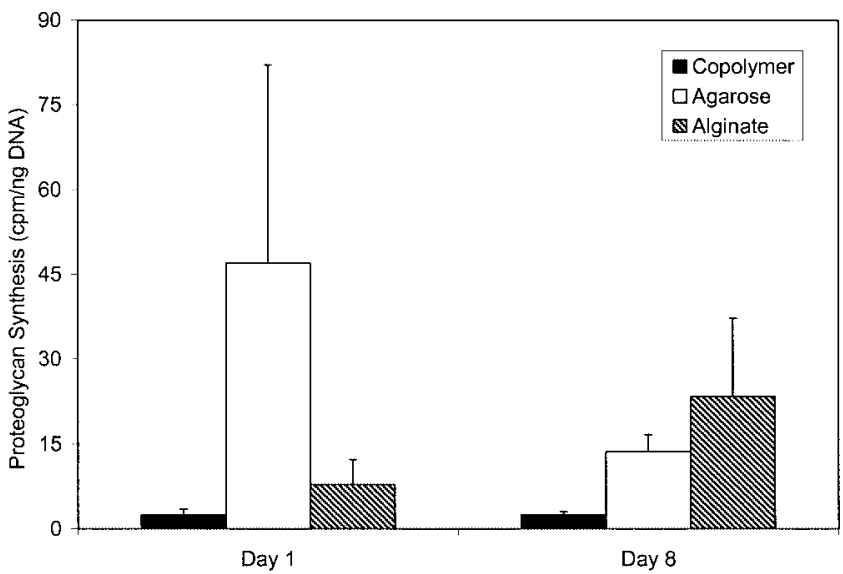

(A)

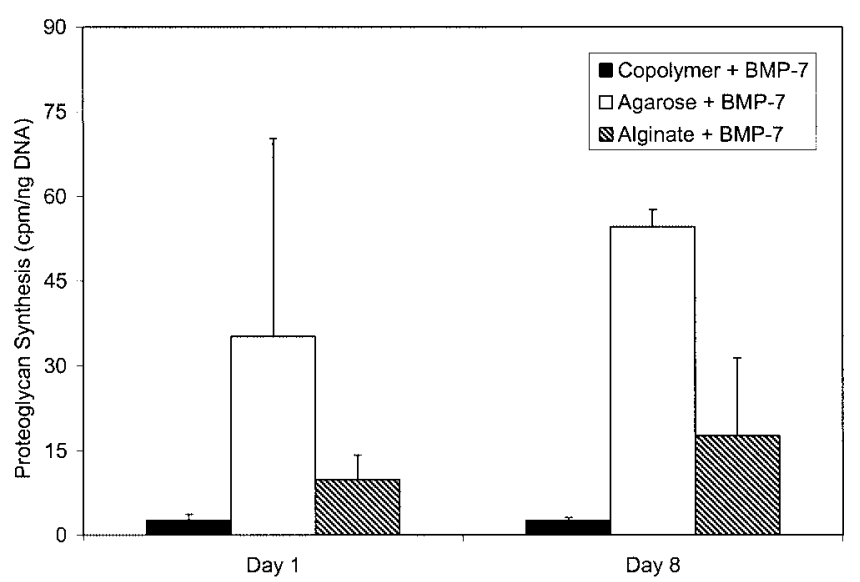

(B)

Figure 3. Chondrocytic proteoglycan synthesis with $\mathrm{P}(\mathrm{PF}-$ co-EG), agarose, and alginate hydrogels after 1 and 8 days of culture in normal DMEM/F12 media (A) and DMEM/F12 media with $100 \mathrm{ng} / \mathrm{mL}$ BMP-7 (B). Proteoglycan synthesis was reduced in the $\mathrm{P}(\mathrm{PF}-\mathrm{co}-\mathrm{EG})$ hydrogels compared with the two control hydrogels and the addition of BMP-7 did not significantly alter this response. The mean and standard deviation $(n=3)$ are reported.

Final studies investigated the effects of BMP-7 concentration (from 1 to $100 \mathrm{ng} / \mathrm{mL}$ ) on extracellular matrix production when cultured in $\mathrm{P}(\mathrm{PF}-\mathrm{co}-\mathrm{EG})$ hydrogels. Results showed that increasing BMP-7 concentration improved cell viability, with the highest levels of BMP-7 $(100 \mathrm{ng} / \mathrm{mL})$ resulting in a statistically significant $(p<0.039)$ increase in cell viability over normal media [Fig. 5(A)]. At this level of BMP-7 concentration, however, proteoglycan synthesis was found to be significantly lower than when cultured in normal media $(p<0.010)$, with a general trend of increasing BMP-7 concentrations resulting in decreased proteoglycan synthesis [Fig. 5(B)].

\section{DISCUSSION}

Articular cartilage disruption due to trauma is a significant clinical concern because of the paucity of inherent healing by the tissue. Clinical treatments often lead to the formation of fibrocartilage and are associated with discomfort, pain, and lack of mobility in the articulating joint. We have investigated the use of a temperature-sensitive hydrogel for the delivery of articular chondrocytes and morphogens to the defect site as a means for cartilage repair. The objectives of this work were to determine the effects of (1) hydrogel components on chondrocyte phenotype, (2) hydrogel suspension culture on chondrocyte phenotype, and (3) BMP-7 augmentation of chondrocyte phenotype within hydrogel suspension culture.

The results of this initial study show that the P(PFco-EG) hydrogels show promise as a delivery vehicle for chondrocyte transplantation. The initial study investigated the effect of the components necessary for hydrogel fabrication on the proliferation and function of the entrapped chondrocytes. Results showed that the addition of either the copolymer or glycerol to the culture media did not cause a decrease in proteoglycan synthesis [see Fig. 1(A,B)]. The successful augmentation of the cell culture media with glycerol was cautiously expected, because the use of glycerol for cryopreservation and specifically cartilage graft preservation, has been previously examined. ${ }^{12-14}$

The next series of experiments sought to compare the chondrocyte's phenotypic expression of proteoglycans and type II collagen when cultured in the P(PFco-EG) hydrogels to that seen in the control alginate and agarose hydrogels. Both alginate and agarose hydrogels have been studied as suspension culture materials for chondrocytes, and generally promote in vivo chondrocyte function despite their in vitro environment. ${ }^{6,15-19}$ Results showed that chondrocyte proliferation within the P(PF-co-EG) hydrogels was comparable to that in agarose and alginate hydrogels (see Fig.

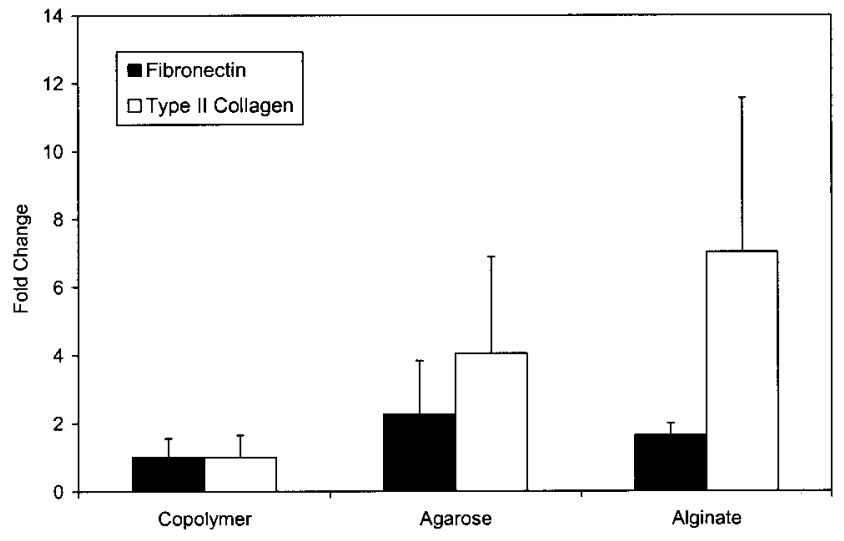

Figure 4. Type II collagen and fibronectin synthesis by bovine articular chondrocytes after 8 days of culture in $\mathrm{P}(\mathrm{PF}-\mathrm{co}-\mathrm{EG})$, agarose, and alginate hydrogels, as measured by quantitative RT-PCR. Type II collagen synthesis by chondrocytes cultured in P(PF-co-EG) hydrogels was $14 \%$ of that seen in alginate hydrogels and $25 \%$ of that seen in agarose hydrogels. The mean and standard deviation $(n=3)$ are reported. 


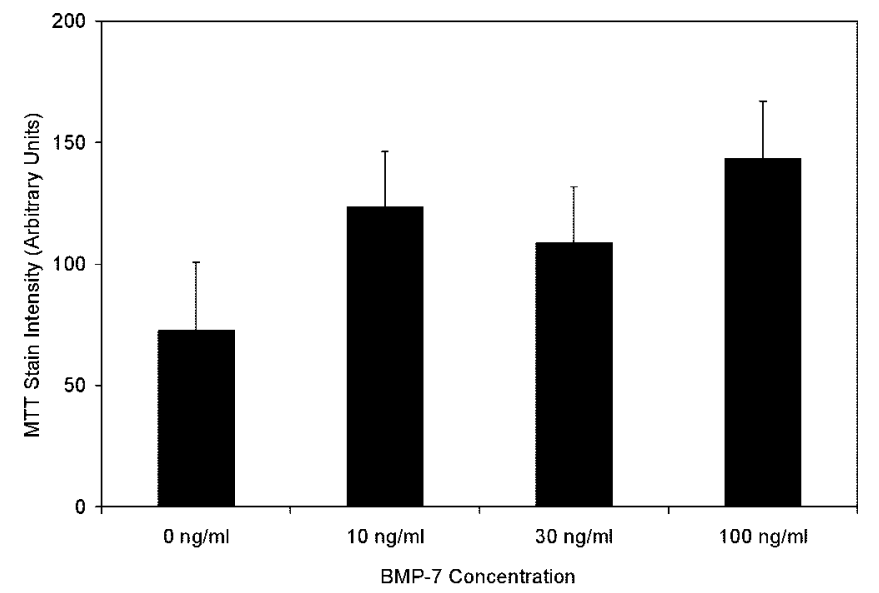

(A)

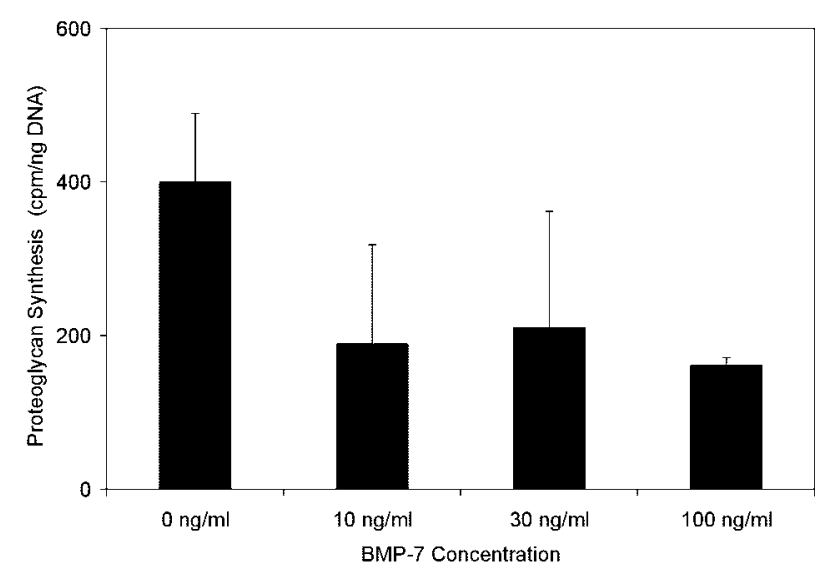

(B)

Figure 5. The effect of BMP-7 addition on chondrocyte proliferation and proteoglycan synthesis when cultured within $\mathrm{P}(\mathrm{PF}-\mathrm{co}-\mathrm{EG})$ hydrogels. Results showed the expected increase in chondrocyte proliferation and decrease in proteoglycan synthesis as BMP-7 concentration was increased from 0 to $100 \mathrm{ng} / \mathrm{mL}$. The mean and standard deviation $(n=$ 3) are reported.

2). Furthermore, the results confirm that chondrocytes cultured within the $\mathrm{P}(\mathrm{PF}-\mathrm{co}-\mathrm{EG})$ hydrogels produce significant levels of both proteoglycans and type II collagen. However, proteoglycan synthesis at both day 1 and day 8 as well as the type II collagen synthesis at day 8 were significantly lower than that in the control settings [see Figs. 3(A) and 4]. Specifically, type II collagen synthesis was $14 \%$ of that seen in alginate hydrogels and $25 \%$ of that seen in agarose hydrogels. The addition of BMP-7 to these cultures did not significantly alter the proteoglycan synthesis, regardless of the hydrogel used for chondrocyte encapsulation [Fig. 3(B)].

The well-known role of BMPs in skeletal tissue formation, repair, and regeneration led us to investigate whether BMP-7 could improve chondrocyte viability in the P(PF-co-EG) hydrogels. ${ }^{7,20-22}$ Thus, the effects of BMP-7 concentration on chondrocyte viability and proteoglycan synthesis were examined. Here, the re- sults show an expected increase in chondrocyte proliferation with increasing BMP-7 concentration [Fig. 5(A)]. Furthermore, the chondrocyte proliferation was confirmed by the decrease in proteoglycan synthesis with increasing BMP-7 concentration [Fig. 5(B)], as gene expression shifted from an extracellular matrix production scheme to a cellular division scheme. Thus, the results presented herein show the BMP-7 is effective in expanding chondrocyte population when cultured within P(PF-co-EG) hydrogels. Additional increases in extracellular matrix production would likely require continued incubation of the expanded chondrocyte population. The results suggest that the $\mathrm{P}(\mathrm{PF}-\mathrm{co}-\mathrm{EG})$ hydrogels are a promising construct for chondrocyte encapsulation, suspension culture, and subsequent delivery to articular cartilage defects. These purely synthetic constructs form without the use of a crosslinking agent and thus are able to sustain a significant level of articular chondrocyte proliferation and function.

\section{CONCLUSION}

We have developed a synthetic, temperature-responsive polymer system suitable for chondrocyte delivery to articular cartilage defects. This system, based on the copolymer $\mathrm{P}(\mathrm{PF}-\mathrm{co}-\mathrm{EG})$, gels at $37^{\circ} \mathrm{C}$, entrapping the suspended chondrocytes within the hydrogel matrix. Results indicated the components involved in the hydrogel fabrication do not significantly reduce the chondrocyte's phenotypic expression of proteoglycans. Chondrocyte viability was confirmed in the $\mathrm{P}$ (PF-co-EG) hydrogels. The addition of the morphogen BMP-7 increased chondrocyte proliferation within the hydrogels in a dose-dependent manner. Thus, the results show that these P(PF-co-EG) hydrogels are viable biomaterials for the engineering of articular cartilage grafts.

This work was supported by Lawrence Ellison Chair Endowment for Musculoskeletal Molecular Biology and Regenerative Medicine as well as a grant from the National Institutes of Health to A. G. Mikos.

\section{References}

1. Broom ND, Oloyede A. The importance of physicochemical swelling in cartilage illustrated with a model hydrogel system. Biomaterials 1998;19(13):1179-1188.

2. Lu L, Zhu X, Valenzuela RG, Currier BL, Yaszemski MJ. Biodegradable polymer scaffolds for cartilage tissue engineering. Clin Orthop 2001;391 Suppl:S251-S270.

3. Buckwalter JA. Articular cartilage injuries. Clin Orthop 2002; 402:21-37.

4. Horas U, Pelinkovic D, Herr G, Aigner T, Schnettler R. Autologous chondrocyte implantation and osteochondral cylinder transplantation in cartilage repair of the knee joint: a prospec- 
tive, comparative trial. J Bone Joint Surg Am 2003;85-A(2):185192.

5. Glowacki J, Trepman E, Folkman J. Cell shape and phenotypic expression in chondrocytes. Proc Soc Exp Biol Med 1983;172(1): 93-98.

6. Benya PD, Shaffer JD. Dedifferentiated chondrocytes reexpress the differentiated collagen phenotype when cultured in agarose gels. Cell 1982;30(1):215-224.

7. Luyten FP, Yu YM, Yanagishita M, Vukicevic S, Hammonds RG, Reddi AH. Natural bovine osteogenin and recombinant human bone morphogenetic protein- $2 \mathrm{~B}$ are equipotent in the maintenance of proteoglycans in bovine articular cartilage explant cultures. J Biol Chem 1992;267(6):3691-3695.

8. Behravesh E, Shung AK, Jo S, Mikos AG. Synthesis and characterization of triblock copolymers of methoxy poly(ethylene glycol) and poly(propylene fumarate). Biomacromolecules 2002;3(1):153-158.

9. Behravesh E, Jo S, Zygourakis K, Mikos AG. Synthesis of in situ cross-linkable macroporous biodegradable poly(propylene fumarate-co-ethylene glycol) hydrogels. Biomacromolecules 2002;3(2):374-381.

10. Hogg RV, Ledolter J. Applied statistics for engineers and physical scientists. New York: Macmillan; 1992. 472 p.

11. Neter J, Kutner MH, Nachtsheim CJ, Wasserman W. Applied linear statistical models. Boston: WCB McGraw-Hill; 1996.

12. Gole MD, Poulsen D, Marzo JM, Ko SH, Ziv I. Chondrocyte viability in press-fit cryopreserved osteochondral allografts. J Orthop Res 2004;22(4):781-787.

13. Malinin TI, Mnaymneh W, Lo HK, Hinkle DK. Cryopreservation of articular cartilage. Ultrastructural observations and long-term results of experimental distal femoral transplantation. Clin Orthop 1994;303:18-32.
14. Marco F, Leon C, Lopez-Oliva F, Perez AJ, Sanchez-Barba A, Lopez-Duran Stern L. Intact articular cartilage cryopreservation. In vivo evaluation. Clin Orthop 1992;283:11-20.

15. Rahfoth B, Weisser J, Sternkopf F, Aigner T, von der Mark K, Brauer R. Transplantation of allograft chondrocytes embedded in agarose gel into cartilage defects of rabbits. Osteoarthritis Cartilage 1998;6(1):50-65.

16. Guo JF, Jourdian GW, MacCallum DK. Culture and growth characteristics of chondrocytes encapsulated in alginate beads. Connect Tissue Res 1989;19(2-4):277-297.

17. Elisseeff JH, Lee A, Kleinman HK, Yamada Y. Biological response of chondrocytes to hydrogels. Ann NY Acad Sci 2002; 961:118-122.

18. Miralles G, Baudoin R, Dumas D, Baptiste D, Hubert P, Stoltz JF, Dellacherie E, Mainard D, Netter P, Payan E. Sodium alginate sponges with or without sodium hyaluronate: in vitro engineering of cartilage. J Biomed Mater Res 2001;57(2):268278.

19. Wong M, Siegrist M, Gaschen V, Park Y, Graber W, Studer D. Collagen fibrillogenesis by chondrocytes in alginate. Tissue Eng 2002;8(6):979-987.

20. Lietman SA, Yanagishita M, Sampath TK, Reddi AH. Stimulation of proteoglycan synthesis in explants of porcine articular cartilage by recombinant osteogenic protein-1 (bone morphogenetic protein-7). J Bone Joint Surg Am 1997;79(8):1132-1137.

21. Reddi AH. Role of morphogenetic proteins in skeletal tissue engineering and regeneration. Nat Biotechnol 1998;16(3):247252.

22. Nakashima M, Reddi AH. The application of bone morphogenetic proteins to dental tissue engineering. Nat Biotechnol 2003;21(9):1025-1032. 